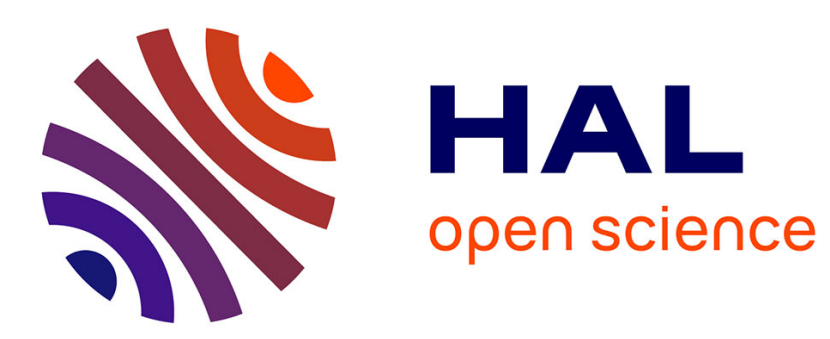

\title{
Free deconvolution for OFDM MultiCell SNR detection
} Romain Couillet, Merouane Debbah

\section{To cite this version:}

Romain Couillet, Merouane Debbah. Free deconvolution for OFDM MultiCell SNR detection. PIMRC 2008, Sep 2008, France. 5 p. hal-00328156

\section{HAL Id: hal-00328156 \\ https://hal-centralesupelec.archives-ouvertes.fr/hal-00328156}

Submitted on 9 Oct 2008

HAL is a multi-disciplinary open access archive for the deposit and dissemination of scientific research documents, whether they are published or not. The documents may come from teaching and research institutions in France or abroad, or from public or private research centers.
L'archive ouverte pluridisciplinaire HAL, est destinée au dépôt et à la diffusion de documents scientifiques de niveau recherche, publiés ou non, émanant des établissements d'enseignement et de recherche français ou étrangers, des laboratoires publics ou privés. 


\section{Free deconvolution for OFDM MultiCell SNR detection}

\author{
Romain Couillet \\ NXP Semiconductors, Supelec \\ 505 Route des Lucioles \\ 06560 Sophia Antipolis, France \\ Email: romain.couillet@nxp.com
}

\author{
Mérouane Debbah \\ Alcatel-Lucent Chair, Supelec \\ Plateau de Moulon, 3 rue Joliot-Curie \\ 91192 Gif sur Yvette, France \\ Email: merouane.debbah@supelec.fr
}

\begin{abstract}
In this paper, a new blind OFDM multicell detection method is proposed to determine the number of base stations in a cellular system. Using recent results of free deconvolution, the algorithm enables the terminal to count the number of surrounding base stations as well as the received power using only a limited number of snapshots. This is in sharp contrast with classical asymptotic blind techniques and a theoretical analysis is proposed to study the impact of frequency selectivity and the number of receive/transmit antennas. Simulations are provided to sustain the theoretical claims and comparisons are provided with classical techniques.
\end{abstract}

\section{INTRODUCTION}

The ever increasing demand of high data rate has pushed system designers to exploit the wireless channel medium to the smallest granularity. In this respect, OFDM (Orthogonal Frequency Division Multiplexing) modulation has been chosen as the next common standard for most wireless technologies (e.g. Wi-Max [3], LTE [2]). OFDM is known to convert a frequency selective fading channel into a set of flat fading channels [12], providing therefore a high flexibility in terms of power and rate allocation. Due to this flat fading nature, OFDM suffers however from a lack of diversity and is severely interfered in multi-cell systems. This requires either system level interference management (with proper multi-cell scheduling) solutions from a network MIMO point of view ([16],[4]). In order to design a viable network solution for OFDM systems, a key parameter that needs to be estimated is the Signal to Interference plus Noise (SINR) ratio that is the power of the base station dedicated to the terminal over the cumulated power of the interfering base stations and the background noise power. Ideally, one needs to access the respective Signal to Noise Ratio of every cell, defined as $\mathrm{SNR}_{k}$, for base station $k$ (the ratio between the power of the signal received by the terminal that originated from transmitter $k$ and the noise level $\sigma^{2}$ ). Usually, this difficult problem of source separation is treated with respect to the signal statistics [13],[14],[15] (through second order statistics and constraints on the input signal), with a hypothesis of a high number of received snapshots. However, in practice, this hypothesis can never be met due to the high mobility of the users.

In this paper, using results on free deconvolution, we show that, in a downlink OFDM environment, one can have access to the power $P_{k}$ of every base station, $k \in[1, M]$, where

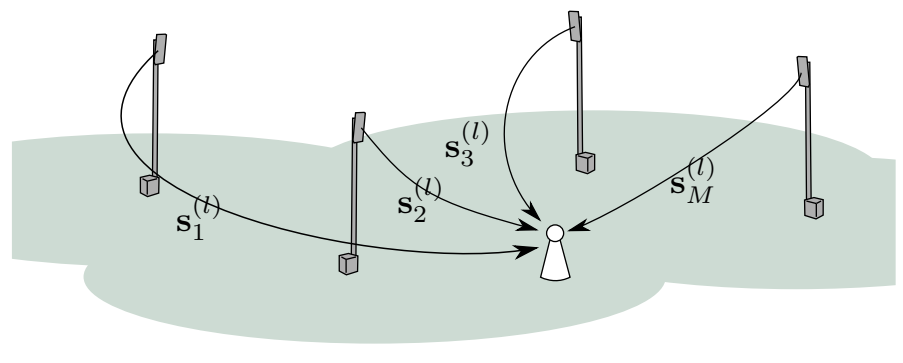

Fig. 1. System Model

$M$ is the assumed maximum number of relevant cells in the network, with no need for any prior knowledge on the input signal constellations (apart from their second order statistics) and using a limited number of received samples (compared to the FFT size). Interestingly, one can derive all the $S N R_{k}$ 's as the ratios $P_{k} / \sigma^{2}$ and also count the number $N_{B} \leq M$ of effective surrounding cells. To the authors knowledge, no previous contribution has ever considered this OFDM multiple SNR detection setting. The work makes extensive use of free deconvolution techniques. Those techniques were initially used in [5] to derive the respective powers of users in a Code Division Multiple Access (CDMA) network.

The paper is structured as follows: In section II, we introduce the multicell environment model. In section III, we review classical methods used to derive SINR in OFDM environments. In section IV, we provide the algorithm to determine the per-cell SNR. A discussion on the gains and limitations of this novel method is then carried out in section $\mathrm{V}$, before we show the simulation results that sustain the theoretical claims. Finally, in section VII we draw our conclusions.

\section{Downlink Model}

In the following, boldface symbols $\mathbf{x}$ represent vectors, $\mathbf{X}$ type characters are used for matrices $\left(\mathbf{I}_{N}\right.$ is the identity matrix of size $N$ ) with Hermitian transpose $(\cdot)^{H} \cdot \operatorname{diag}(\mathbf{x})$ turns the vector $\mathrm{x}$ into a diagonal matrix. $C_{n}^{k}$ is used for binomial coefficients. $\operatorname{tr}_{N}(\cdot)$ will be used for the normalized trace of an $N \times \cdot$ matrix and $\mathbb{E}[\cdot]$ is the expectation symbol.

Let us consider, as depicted in figure II, a set of $N_{B}$ base stations and one UE (User equipment) with $N_{r}=1$ receiving 
antenna working under a size $N$ FFT OFDM modulation. Let us also fix an integer $M$ meant to count all the base stations (ideally $N_{B} \leq M$ ). In the following, we shall actually only deal with $M$ and forget about $N_{B}$, considering then that we have $M$ base stations, some of which could be of null power. The link between the UE and the base station $k$ is a fastfading complex Gaussian channel vector $\mathbf{h}_{k}$ of size $N$ coupled with a slow-fading path loss $L_{k}=1 / P_{k}$ with $P_{k}$ the power received at the UE from data of base station $k$. The UE also suffers Additive White Gaussian Noise (AWGN) of power $\sigma^{2}$. The base station $k$ sends at time $l$ the OFDM symbol $\mathbf{s}_{k}^{(l)}=\left(s_{1 k}^{(l)}, \ldots, s_{N k}^{(l)}\right)^{T}$ that we suppose standard Gaussian (ie, with zero mean and unit variance) under AWGN noise $\sigma \mathbf{n}^{(l)}$, with $\mathbf{n}^{(l)}=\left(n_{1}^{(l)} \ldots, n_{N}^{(l)}\right)^{T}$ also a standard Gaussian vector. Therefore, the received signal vector $\mathbf{y}^{(l)}=\left(y_{1}^{(l)}, \ldots, y_{N}^{(l)}\right)^{T}$ at time instant $l$ reads

$$
\mathbf{y}^{(l)}=\sum_{k=0}^{M-1} P_{k}^{\frac{1}{2}} \mathbf{D}_{k} \mathbf{s}_{k}^{(l)}+\sigma \mathbf{n}^{(l)}
$$

with $\mathbf{D}_{k}=\operatorname{diag}\left(\mathbf{h}_{k}\right)=\operatorname{diag}\left(\left[h_{k 1} \ldots h_{k N}\right]\right)$.

This summation over the $M$ cells can be rewritten

$$
\mathbf{y}^{(l)}=\mathbf{H P}^{\frac{1}{2}} \boldsymbol{\theta}^{(l)}+\sigma \mathbf{n}^{(l)}
$$

with $\boldsymbol{\theta}^{(l)}$, the size- $M N$ vector $\boldsymbol{\theta}^{(l)}=\left(\mathbf{s}_{1}^{(l)}, \ldots, \mathbf{s}_{M}^{(l)}\right)^{T}$

$\mathbf{H}$ is the concatenated matrix of size $N \times M N$ of the matrices $\mathbf{D}_{k}, k \in[1, M]$

$$
\mathbf{H}=\left[\begin{array}{ccccccc}
h_{11} & \cdots & 0 & \cdots & h_{M 1} & \cdots & 0 \\
\vdots & \ddots & \vdots & \cdots & \vdots & \ddots & \vdots \\
0 & \cdots & h_{2 N} & \cdots & 0 & \cdots & h_{M N}
\end{array}\right]
$$

and $\mathbf{P}$ the diagonal matrix of width $N M$

$$
\mathbf{P}=\operatorname{diag}\left(\left[P_{1} P_{2} \cdots P_{M}\right]\right) \otimes \mathbf{I}_{N}
$$

where the symbol $\otimes$ stands for the Kronecker product.

Let us now assume that the $M$ channels are slowly varying, so that we can concatenate $L$ samples $\mathbf{y}^{(l)}(L=1, \ldots, L)$ into an $N \times L$ matrix $\mathbf{Y}=\left[\mathbf{y}^{(\mathbf{1})} \cdots \mathbf{y}^{(\mathbf{L})}\right]$ and have the more general matrix product

$$
\mathbf{Y}=\mathbf{H} \mathbf{P}^{\frac{1}{2}} \mathbf{\Theta}+\sigma \mathbf{N}
$$

where again $\Theta$ and $\mathbf{N}$ are respectively the $M N \times L$ and $N \times L$ concatenation matrices of the $L$ sampling time instants of $\boldsymbol{\theta}^{(\cdot)}$ and $\mathbf{n}^{(\cdot)}$ and the entries of $\mathbf{H}$ are fixed over the $L$ channels uses.

This then imposes the minimum of all $M$ channel coherence times to be greater than $L T_{s}$ with $T_{s}$ the OFDM symbol period.

\section{Classical Power Detection}

Usual power detectors consider the second and higher order statistics of the received signals. This consists in computing the following empirical moments: $\left(\frac{1}{L} \mathbf{Y} \mathbf{Y}^{H}\right)^{k}$. These techniques work well when $L$, the number of samples, go to infinity while $N$, the size of the observation vector is finite or at least

\begin{tabular}{c|c|c}
\hline \hline \multicolumn{3}{|c}{$N=256, \mathbf{P}=\left\{P_{1}, P_{2}, P_{3}\right\}=\{4,2,1\}$} \\
\hline \hline$L$ & Estimated $\tilde{\mathbf{P}}[$ our algorithm] & $\|\mathbf{P}-\tilde{\mathbf{P}}\|_{2}$ \\
\hline 512 & $\{6.24,2.3,-1.5\}[\{4.14,1.73,1.16\}]$ & 11.33 \\
1024 & $\{5.08,2.56,-0.7\}[\{3.99,2.02,1.02\}]$ & 4.44 \\
2048 & $\{4.52,2.69,-0.2\}[\{4.11,1.89,1.03\}]$ & 2.2 \\
4096 & $\{4.2,2.65,0.18\}[\{3.98,1.95,1.03\}]$ & 1.13 \\
8192 & $\{4.1,2.28,0.58\}[\{4.1,1.8,1.11\}]$ & 0.27 \\
16384 & $\{3.97,2.42,0.89\}[\{4.06,1.81,1.35\}]$ & 0.19 \\
32768 & $\{4.07,1.95,0.99\}[\{3.93,1.88,1.16\}]$ & 0.01 \\
\hline
\end{tabular}

Fig. 2. Classical moment-based method

when the ratio $N / L$ tends to zero when both $N$ and $L$ go to infinity. Indeed, when $L$ grows, $\left(\frac{1}{L} \mathbf{Y} \mathbf{Y}^{H}-\sigma^{2} \mathbf{I}_{N}\right)^{k}$ tends to $\mathbb{E}\left[\left(\mathbf{H P} \mathbf{H}^{H}\right)^{k}\right]$, which is related to the power expression needed.

As a consequence, one can retrieve the values of the $P_{k}$ 's (as will be proved in section IV) directly from the normalized traces of $\frac{1}{L}\left(\mathbf{Y} \mathbf{Y}^{H}-\sigma^{2} \mathbf{I}_{N}\right)^{k}$ when $L$ is large compared with $N$. However, in practice, this case is rarely met due to mobility of the UE. In fact, we wish $N$ to be fairly large (such that the ratio between the $N$-subcarrier bandwidth over the coherence bandwidth is large) while $L$ is limited by the channel coherence time. Therefore, even if $N$ and $L$ are large, the problem fall in a situation where the ratio $N / L$ is non trivial (ie, not close to 0) so that expectation-based methods are far from accurate. In this context, the previous classical method does not work since the expectation taken for large $L$ is no more valid when $N$ grows along with $L$. This is shown in table (2) that uses the same algorithm as described in section (IV-A) based on the moments of $\frac{1}{L}\left(\mathbf{Y} \mathbf{Y}^{H}-\sigma^{2} \mathbf{I}_{N}\right)$ (instead of the moments of $\frac{1}{L} \mathbf{H P H}{ }^{H}$ for which comparative results, based on the algorithm of section IV, are presented between brackets).

For a deeper analysis of those classical techniques, please refer to [17]. In this contribution, we shall also use momentsbased techniques but in the light of the recent work on Random Matrix Theory (RMT) [6] and Free Deconvolution [5], that are briefly introduced in the following.

\section{Application of Free Deconvolution to MULTIPLE SNR DETECTION}

In order to recover the $P_{i}$ values, as suggested in the previous section, one needs to have access to the entries of $\mathbf{H P H}^{H}$. As shall be shown later, one needs only to have access to the eigenvalue distribution of $\mathbf{H P H}{ }^{H}$. This distribution is called in the RMT context the empirical distribution of the matrix $\mathbf{H P H}{ }^{H}$ and is denoted $\mu_{\mathbf{H P H}}{ }^{H}$. For those distributions, we associate free moments $M_{k}$ of order $k$ defined as $M_{k}=\mathbb{E}\left[\operatorname{tr}_{N}\left(\mathbf{H P H} \mathbf{H}^{H}\right)^{k}\right]$. When an $N \times N$ random matrix $\mathbf{A}$ is a standard Wishart matrix, which can be written $\mathbf{A}=\frac{1}{L} \mathbf{X X}^{H}$, with $\mathbf{X}$ an $N \times L$ standard Gaussian random matrix (that is a matrix with standard iid Gaussian entries), then its empirical distribution is the Marchenko-Pastur law [6] that we denote $\mu_{\eta_{c}}$, with $c=N / L$. Those Wishart matrices 
have a generalized version in which the column entries of $\mathbf{X}$ are correlated through a covariance matrix $\Sigma_{\mathbf{X}}$. Recent work on Free Probability [7] and RMT [5], [9] have provided several tools to derive the empirical distributions of the sum, product... of random matrices. In particular, when the matrix at hand is of the information plus noise type (those random matrices are deeply studied in [5]), then it is possible to access the empirical distribution of the information signal given the empirical distribution of the received information plus noise signal. This is the main result that we use in this work, which enters the general framework of free deconvolution. We shall use in the following the symbols $\boxplus, \boxminus, \otimes$ and $\nabla$ respectively to retrieve the empirical distribution of the sum, difference, product and inverse of two random matrices. For instance

$$
\begin{aligned}
\mu_{\mathbf{A}+\mathbf{B}} & =\mu_{\mathbf{A}} \boxplus \mu_{\mathbf{B}} \\
\mu_{\mathbf{C}} & =\mu_{\mathbf{A}} \nabla \mu_{\mathbf{B}}
\end{aligned}
$$

with $\mathbf{C}$ such that $\mathbf{A}=\mathbf{C B}$.

In our problem, described in the form of model (5), it turns out that the $N \times N$ matrix $\frac{1}{L} \mathbf{Y} \mathbf{Y}^{H}$ is an information plus noise matrix with $\mathbf{N}$ a Gaussian random matrix (then $\frac{1}{L} \mathbf{N} \mathbf{N}^{H}$ is a Wishart matrix). Therefore, for large $(N, L)$ values, one can derive the empirical distribution of $\frac{1}{L} \mathbf{H} \mathbf{P}^{\frac{1}{2}} \boldsymbol{\Theta} \Theta^{H} \mathbf{P}^{\frac{1}{2}} \mathbf{H}^{H}$

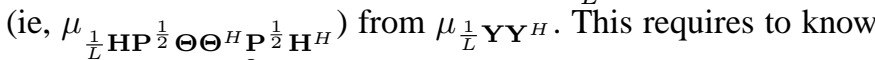
the noise level $\sigma^{2}$ and reads [5]

$$
\mu_{\frac{1}{L} \mathbf{H} \mathbf{P}^{\frac{1}{2}} \Theta^{H} \mathbf{P}^{\frac{1}{2}} \mathbf{H}^{H}}=\left(\left(\mu_{\frac{1}{L} \mathbf{Y} \mathbf{Y}^{H}} \nabla \mu_{\eta_{c}}\right) \boxminus \delta_{\sigma^{2}}\right) \bigotimes \mu_{\eta_{c}}
$$

where $c=N / L$ since the noise matrix is $N \times L$.

Also, the matrix $\Theta$ in equation (5) was made such that its random entries are standard Gaussian and independent. Therefore $\frac{1}{L} \mathbf{P}^{\frac{1}{2}} \mathbf{H}^{H} \mathbf{H} \mathbf{P}^{\frac{1}{2}} \boldsymbol{\Theta} \Theta^{H}$ is a generalized Wishart matrix with covariance $\mathbf{P}^{\frac{1}{2}} \mathbf{H}^{H} \mathbf{H} \mathbf{P}^{\frac{1}{2}}$.

As such, $\mu_{\mathbf{P}^{\frac{1}{2}} \mathbf{H}^{H} \mathbf{H P}^{\frac{1}{2}}}$ can be recovered from

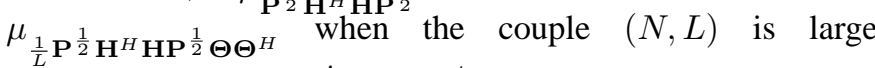
with a constant ratio $c^{\prime}=M N / L$ ( $M$ is constant) [5]

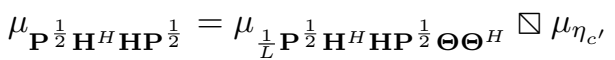

Note that the left expression of equation (8) is slightly different from the desired expression in the right part of equation (9). Still, thanks to the trace property, we have the trivial link [6]

$\mu_{\frac{1}{L} \mathbf{P}^{\frac{1}{2}} \mathbf{H}^{H} \mathbf{H} \mathbf{P}^{\frac{1}{2}} \boldsymbol{\Theta} \Theta^{H}}=\frac{1}{M} \mu_{\frac{1}{L} \mathbf{H} \mathbf{P}^{\frac{1}{2}} \Theta_{\Theta} \Theta^{H} \mathbf{P}^{\frac{1}{2}} \mathbf{H}^{H}}+\left(1-\frac{1}{M}\right) \delta_{0}$

Finally, we similarly connect the left part of equation (9) to $\mu_{\mathbf{H P H}^{H}}$ through

$$
\mu_{\mathbf{P}^{\frac{1}{2}} \mathbf{H}^{H} \mathbf{H P}^{\frac{1}{2}}}=\frac{1}{M} \mu_{\mathbf{H P H}^{H}}+\left(1-\frac{1}{M}\right) \delta_{0}
$$

As a consequence, we have shown that, thanks to the link between their associated empirical distributions, the free moments $d_{k}=\mathbb{E}\left[\operatorname{tr}{ }_{N}\left(\mathbf{H P H} \mathbf{H}^{H}\right)^{k}\right]$ can be retrieved from the free moments $m_{k}=\mathbb{E}\left[\operatorname{tr}_{N}\left(\frac{1}{L} \mathbf{Y} \mathbf{Y}^{H}\right)^{k}\right]$. Quite surprisingly, it is shown [8] that for any of the free classical operations (additive convolution, deconvolution...), the ensemble of the first $k$ moments of the operation result can be exactly recovered from the ensemble of the $k$ first moments of the operands (and viceversa). This substancially reduces the computational effort.

The details of how to recover the moments $d_{k}$ from the moments $m_{k}$ as well as fundamentals of Random Matrix Theory and Free Deconvolution are provided in [17].

Our interest though is to find the diagonal values of P. Quite remarkably, it turns out that the matrix $\mathbf{H} \mathbf{P} \mathbf{H}^{H}$ is diagonal. Therefore, for large $(N, L)$ couples, we can easily derive all the theoretical free moments $d_{k}$ of the distribution $\mu_{\mathbf{H P H}^{H}}$ [6] since all the $\left(\mathbf{H} \mathbf{P} \mathbf{H}^{H}\right)^{p}$ are diagonal matrices of $(i, j)$ entry

$$
\left\{\left(\mathbf{H P H}^{H}\right)^{p}\right\}_{i j}=\left(\sum_{k=1}^{M} P_{k}\left|h_{k i}\right|^{2}\right)^{p} \delta_{i}^{j}
$$

and then the $p^{t h}$ order moment $d_{p}=\mathbb{E}\left[\operatorname{tr}_{N}\left(\mathbf{H P H} \mathbf{H}^{H}\right)^{p}\right]$ of $\mathbf{H P H}^{H}$ can then be approximated for large $N$ by

$$
d_{p}=\frac{1}{N} \sum_{j=1}^{N}\left(\sum_{k=1}^{M} P_{k}\left|h_{k j}\right|^{2}\right)^{p}
$$

At this point, the $d_{p}$ 's expression (13) contains too many unknowns since, in addition to the $P_{k}$ 's, also the $h_{i j}$ are unknown. In fact, as is discussed in section (V), these are those very unknown $h_{i j}$ that allow for the multiple SNR recovery provided that the channel coherence bandwidth (ie, the frequency range in which the channel frequency responses are correlated) is short compared to the system bandwidth. In the following we therefore discuss the frequency selective scenario under the hypothesis that the ratio coherence bandwidth over system bandwidth is short.

\section{A. Finding the $P_{k}$ 's}

To deduce the cell power values from the moments $d_{p}$, we need to derive independant equations from those $d_{p}$ 's in the variables $\left\{P_{k}, k \in[1, M]\right\}$. Again, as will be discussed in section (V), the channels' (associated to every emitting base station) frequency diversity is the key to provide those equations. Let us then start by deriving, for large $L$, the moments $d_{p}$ as in (13), which, for $p \in[1, M]$, forms a system of $M$ equations in the $M$ unknowns $\left\{P_{k}, k \in[1, M]\right\}$.

In this case, since $N$ is large, (13) can be approximated by

$$
\mathbb{E}_{h}\left(\sum_{k=1}^{M} P_{k}\left|h_{k j}\right|^{2}\right)^{p}=d_{p}
$$

where $\mathbb{E}_{h}$ denotes the expectation over the variables $h_{i j}$.

With $N$ as we chose (ie, much larger than the typical coherence bandwidth size), we then have a high confidence that the channel correlations do not have a strong impact in the final results and then, based on the classical moments of the Rayleigh-distributed variables $\left|h_{k j}\right|$, we can derive $d_{p}$ as

$$
d_{p}=\frac{p !}{2^{2 p}} \sum_{\substack{k_{1}, \ldots, k_{M} \\ \sum_{i} k_{i}=p}} \prod_{i=1}^{M}\left\{\sum_{k=0}^{k_{i}} \frac{(2 k) !\left(2\left[k_{i}-k\right]\right) !}{(k !)^{2}\left(\left[k_{i}-k\right] !\right)^{2}}\right\} P_{i}^{k_{i}}
$$


the proof of formula (15) and further details about the "large $N$ " hyptothesis are provided in [17].

Therefore the system of equations formed by (15) for $p \in[1, M]$ is constituted of multivariate polynomials in $P_{1}, \ldots, P_{M}$. This homogenous symmetric multivariate polynomial system can be rewritten

$$
\sum_{k=1}^{M} P_{k}^{p}=Q_{p}\left(d_{1}, \ldots, d_{p}\right)
$$

for polynomial functions $Q_{k} \in R\left[d_{1}, \ldots, d_{k}\right]$ to be determined.

System (16) is then easier to solve. Its solution, the vector of powers $\left(P_{1}, \ldots, P_{M}\right)$, is unique and corresponds to the $M$ roots (counted with their multiplicities) of the polynomial in $X$ of degree $M$

$$
X^{M}-\Pi_{1} X^{M-1}+\Pi_{2} X^{M-2}-\ldots+(-1)^{M} \Pi_{M}
$$

where the elements $\Pi_{k}$ are related to the $\sum_{j} P_{j}^{i}$ through the Newton-Girard formula [10]

$$
(-1)^{k} k \Pi_{k}+\sum_{i=1}^{k}(-1)^{k+i}\left(\sum_{j=1}^{M} P_{j}^{i}\right) \Pi_{k-i}=0
$$

A thorough and clear study of the particular $M=3$ case is derived in [17], as well as the complete derivations that lead to find the $Q_{k}$ polynomials.

\section{DISCUSSION}

A few points are worth being discussed in the light of the previous study.

First, as already mentioned in the previous sections, our algorithm simplifies to a mere power detector when the number of sampling periods $L$ available is rather large compared to the FFT size $N$. This would be valid either when $L$ is fairly larger than $N$ but this imposes very long accumulations, which is no longer valid for the typical coherence time encountered in OFDM or when $N$ is limited to a very few elements but then ones looses much of the provided information, which will heavily degrade the performance.

A second item is the channel aspect that is of prior importance. Indeed, if only one simulation shot is run (with sufficiently large $N$ and $L$ ), and if the channel is typically very short, then the channel frequency response will be rather flat over the whole bandwidth.

This implies that all the moments of $\mathbf{H P H}{ }^{H}$ will form a correlated system of equations and (16) cannot be derived since equation (15) does not stand anymore. The best we can figure out from this situation is the approximated total power received from all cells.

This is why a short coherence bandwidth (with respect to the total bandwidth) is desired, so to keep (15) true.

If this short coherence bandwidth is not provided, then the scheme can be extended to use multiple antennaes to introduce independent channel realizations. Then, intead of using an $N \times$ $L \mathbf{Y}$ matrix at the reception, we can easily extend the scheme to use an $N N_{r} \times L$ data matrix with $N_{r}$ the number of receive antennaes at the UE.
Cell Power Detection $-\mathrm{SNR}=10 \mathrm{~dB}$

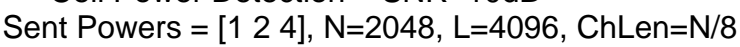

Averaged over 1000 realisations

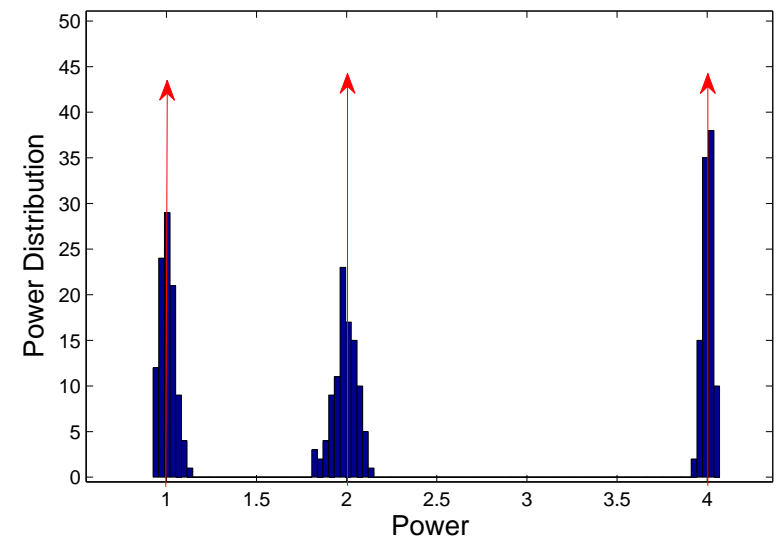

Fig. 3. Cell power detection, $N=512, L=1024$, Averaged over 1000 trials

\section{Simulation AND Results}

In the following, we use the results that were previously derived in the case of a three-cell network that the UE wishes to track. The set of cells studied along this part are of relative powers $P_{1}=4, P_{2}=2, P_{3}=1$.

In a first simulation, in order to increase the performance accuracy, we shall average the estimated $d_{p}$ values on 1000 channel realizations that are exponential decaying OFDM channels of length varying from 1 to $N / 4$ symbols of an OFDM symbol.

In figure (3), we took matrices of $N=512$ FFT size and $L=1024$ sampling periods and a Rayleigh channel of length $N / 8$. A hundred realisations of this process are run. The SNR is $10 \mathrm{~dB}$. Histogram (3) shows that $\left(P_{1}, P_{2}, P_{3}\right)$ is clearly well recovered.

The next experiment consists in estimating the noise level impact on the cell recovery. This is obtained by comparing the $S N R=30 d B$ case to the $S N R=-10 d B$ scenario. Figure (5) provides the results (with 1000 accumulations over 100 trials) and shows that, surprisingly, whatever the noise level (even if it actually perfectly matches one specific cell power), the cell power recovery is substancially the same when $(N, L)$ is large enough.

Also, we need to test the robustness of our algorithm against practical channels and not only theoretical exponential decaying channels. This is done in figure (4) that proposes a comparison between the ideal long channel situation and the 3GPP-Long Term Evolution (LTE) [2] standardized Extended Vehicular A (EVA) and Extended Typical Urban (ETU) channels with characteristics

\begin{tabular}{|c|c|c|}
\hline Channel Type & RMS Delay Spread & Channel Length \\
\hline EVA & $357 n s$ & $N / 27$ \\
ETU & $991 n s$ & $N / 13$ \\
\hline
\end{tabular}

We considered here a mobile handset situation provided with 2 antennaes $N_{r}=2$, working under a size $N=256$-FFT, 


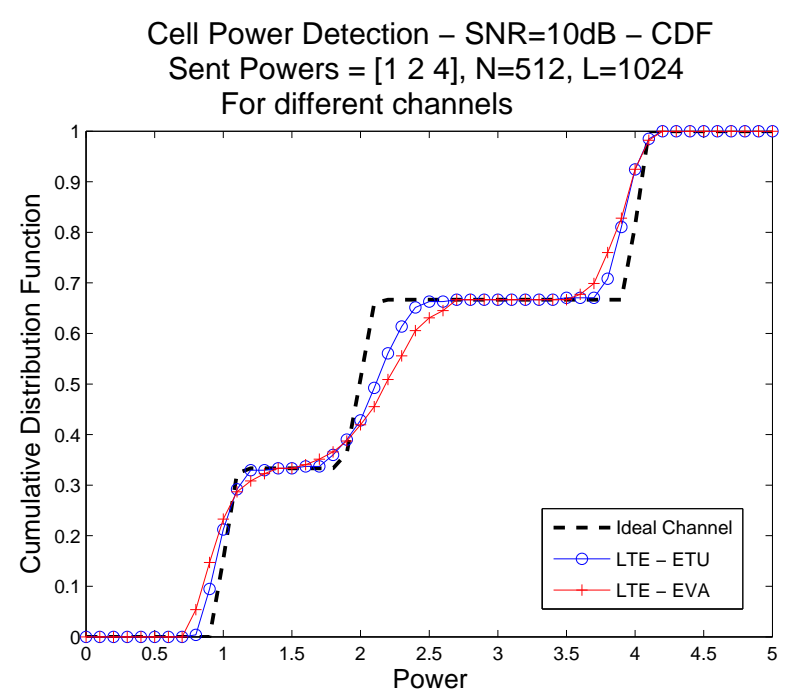

Fig. 4. Cell power detection of LTE short Channels, $L=1024$, Averaged over 1000 trials

with $S N R=10 d B$. $L$ is taken equal to 1024 and the results are averaged over a thousand trials, for fair comparison with the previous results. The Cumulated Distribution Function of the detected power distribution for those channels is provided in (4) that shows a rather good behaviour of both ETU and EVA channels that nonetheless suffer from their being rather short, which leads to less performant results. It is also to be noted that a certain bias in the mean power estimates is introduced in this case.

Quite surprisingly, it turns out that no matter the chosen zero mean unit variance modulation distribution of the input signals $\mathbf{s}^{(l)}$, the results show the same performance. This is a general observation in Free Deconvolution which has not been proven yet. Therefore in our simulations, QPSK modulations showed the exact same behaviour as purely Gaussian distributed signals. Also, we carried out some simulations in which we purposely took $N_{B}$ larger than $M$ (eg, four base stations emitting while only three are assumed). This has fairly bad consequences in that the characteristic polynomial (17) often has non-real solutions. As a consequence, the number of base stations should always be upper bounded.

The Uplink scenario in which a base station wants to determine the powers of multiple UE's in its cell can be equally derived by changing $M$ into the number of potential users in the cell and $N_{r}$ into the number of antennaes at the base station.

\section{CONCLUSION}

In this contribution, we demonstrated a practical way to blindly detect neighboring cells in a distributed OFDM network. Assuming constant transmission of those cells on a fairly large bandwidth (large enough to ensure that the channel coherence bandwidth is small in comparison), we showed that one can blindly determine the individual SNR of every cell. This is particularly suitable to next generation OFDM systems
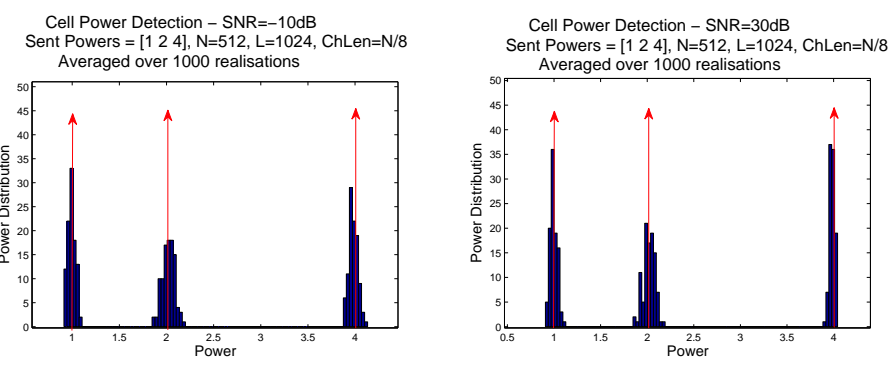

Fig. 5. Cell power detection at $S N R=-10 d B$ (left) and $S N R=30 d B$ (right), $N=512, L=1024$, Averaged over 1000 trials

which aim at reducing the amount of synchronization sequence required to keep track of the neighboring cells.

\section{REFERENCES}

[1] E. Biglieri, J. Proakis, and S. Shamai, "Fading channels: Informationtheoretic and communications aspects," IEEE Trans. on Inf. Theory, vol. 44, no. 6, pp. 2619-2692, Oct 1998.

[2] http://www.3gpp.org/Highlights/LTE/LTE.htm

[3] http://wirelessman.org/

[4] K. Karakayali, G. J. Foschini, R. A. Valenzuela, "Network Coordination for Spectrally Efficient Communications in Cellular Systems", August 2006, IEEE Wireless Communications Magazine (invited).

[5] Ø. Ryan, M. Debbah, "Free deconvolution for signal processing applications", under review, http://arxiv.org/abs/cs.IT/0701025

[6] A. M. Tulino, S. Verdú, "Random Matrix Theory and Wireless Communications", Now Publishers, vol. 1, Issue 1, 2004.

[7] P. Biane, "Free Probability for Probabilists", Quantum Probability Communications, World Scientist, 2003.

[8] Ø. Ryan, M. Debbah, "Multiplicative free convolution and informationplus-noise type matrices", Planned for submission to Journal Of Functional Analysis, 2006, http://www.ifi.uio.no/õyvindry/multfreeconv.pdf

[9] Ø. Ryan, M. Debbah, "Channel Capacity Estimation using Free Probability Theory", 2007, http://arxiv.org/abs/0707.3095

[10] R. Seroul, D. O'Shea, "Programming for Mathematicians", Springer, 2000

[11] M. Hall Jr. "Combinatorial Theory”, John Wiley \& Sons, Inc. New York, NY, USA, 1998

[12] J.A. Bingham, "Multicarrier modulation for data transmission: An idea whose time has come," IEEE Commun. Mag., vol. 28, pp. 5-14, May 1990.

[13] C.B. Papadias, "Globally convergent blind source separation based on a multiuser kurtosis maximization criterion", IEEE Trans. on Signal Processing, Dec. 2000, pp. 3508-3519.

[14] J. Villares, "Symbol-rate Second-Order Blind Channel Estimation in Coded Transmissions", in Proc. ICASSP, Honolulu, Hawaii, April 2007.

[15] Herzet, "Channel Coding Aided Synchronization", seminar presentation, UC Berkeley, April 2007.

[16] E.-V. Belmega, S. Lasaulce, M. Debbah, "Handovers in distributed cognitive cellular networks", To be published

[17] R. Couillet, M. Debbah, "Free deconvolution for Multiple SNR detection in OFDM systems", To be published 